\title{
Megacalycosis: a rare radiological finding
}

\author{
CL Cho *, CK Shiu
}

Hong Kong Med J 2020;26:539.e1-2

https://doi.org/10.12809/hkmj208463

In April 2019, a 22-year-old woman was admitted to Union Hospital, Hong Kong, with right loin pain and fever. She had developed acute cystitis symptoms 1 week prior to the admission and was prescribed a course of oral antibiotic. She had good past health with no history of urinary tract infection. On admission she had a temperature of $39^{\circ} \mathrm{C}$. The abdomen was soft and non-tender. Laboratory tests revealed a normal white cell count of $6.09 \times 10^{9} / \mathrm{L}$ and normal creatinine of $54 \mu \mathrm{mol} / \mathrm{L}$. The $\mathrm{C}$-reactive protein was elevated at $45.5 \mathrm{mg} / \mathrm{L}$. Urine tests revealed a slightly turbid urine with elevated white blood cell of 260 cells $/ \mu \mathrm{L}$. There was no significant growth on urine culture. An urgent contrast computed tomography scan revealed features suggestive of pyelonephritis at the lower pole of the right kidney (Fig 1). There was no obstructive urinary stone. In addition, the imaging demonstrated an atypical right pelvicalyceal system (Fig 2). A course of antibiotic was prescribed with good clinical response. Repeat urine microscopy was normal 8 weeks after the initial presentation. Follow-up computed tomography scan confirmed resolution of right pyelonephritis but the unusual finding of the right pelvicalyceal system remained unchanged (Fig 3).

The radiological features were diagnostic of congenital megacalycosis. The anomaly is characterised by caliectasis with malformation. The classic triangular or conical shape of the renal calyces is replaced by a semilunar configuration.

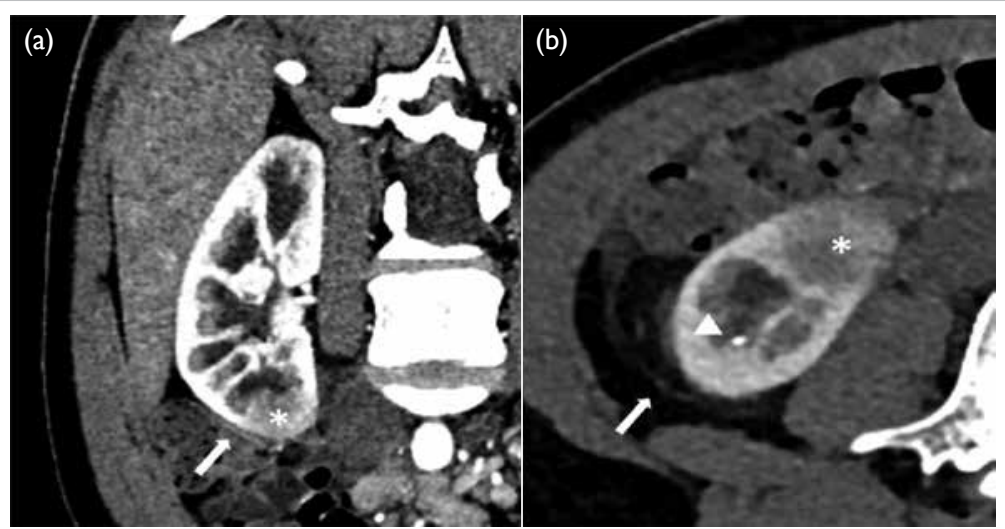

FIG I. Arterial phase contrast-enhanced computed tomography in (a) coronal and (b) axial planes demonstrating focal renal parenchymal swelling and hypoenhancement (asterisk) with associated perinephric fat stranding (arrow) consistent with acute pyelonephritis. Note the presence of a small renal stone (arrowhead) in a dilated calyx

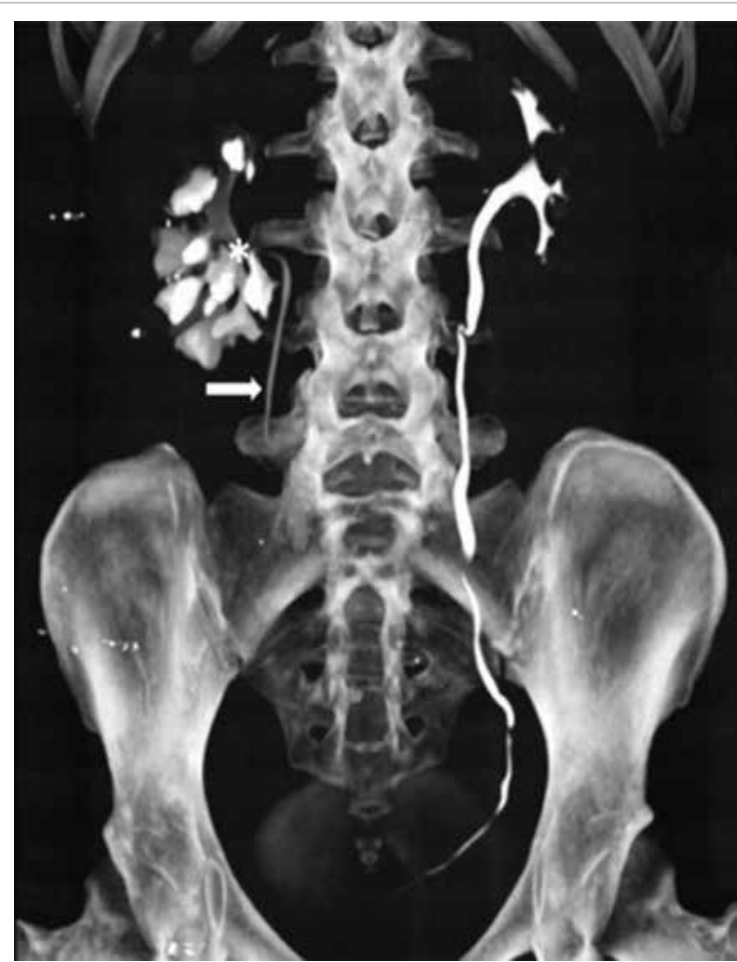

FIG 2. Maximum intensity projection computed tomography urogram showing features of congenital megacalycosis with increased number and dilated, semilunar-shaped calyces in the right kidney. The right renal calyces are well opacified. The right renal pelvis (asterisk) and right ureter (arrow) are normal. Contralateral normal left upper tract urogram is shown for comparison
The pyramids of Malpighi are hypoplastic and the tip of each papilla is flat. The calyces have a rounded appearance with neither fornix nor papillae impressions. Polycalycosis is another feature of the condition and typically 20 to 25 calyces can be identified. The condition is differentiated from obstruction by the finding of a non-dilated renal pelvis, infundibulum, and ureter. The renal cortex was of normal thickness with good concentration of contrast medium in the distended calyces (Figs 2 and 3$)$.

Congenital megacalycosis is a rare condition with approximately 100 cases reported in the literature. The anomaly is found predominantly in males and usually affects only one kidney. The exact pathogenesis remains unclear. ${ }^{1-3}$ The condition is usually asymptomatic and is detected during workup of urinary stone disease or urinary tract 


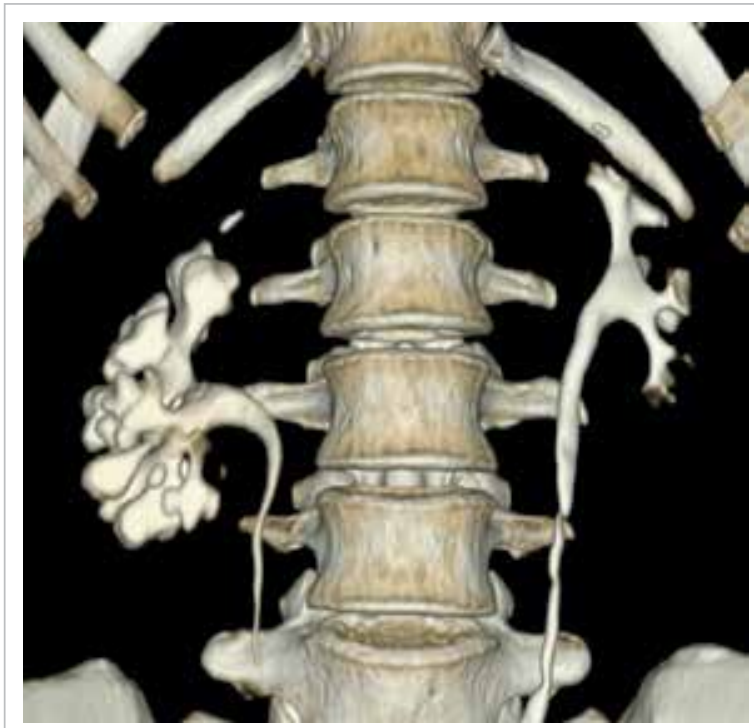

FIG 3. Follow-up computed tomography urogram with three-dimensional volume rendering showing persistent features of congenital megacalycosis with increased number and dilated right renal calyces

\section{Author contributions}

Concept or design: CL Cho.

Acquisition of data: All authors.

Analysis or interpretation of data: All authors.

Drafting of the manuscript: All authors.

Critical revision of the manuscript for important intellectual content: All authors.

All authors had full access to the data, contributed to the study, approved the final version for publication, and take responsibility for its accuracy and integrity.

\section{Conflicts of interest}

The authors have disclosed no conflicts of interest.

\section{Funding/support}

This pictorial medicine paper received no specific grant from any funding agency in the public, commercial, or not-forprofit sectors

\section{Ethics approval}

This study was conducted in accordance with the principles outlined in the Declaration of Helsinki. Verbal consent was obtained for the purpose of case study.

${ }^{1,2}$ CL Cho *, FRCS Ed (Urol), FHKAM (Surgery)

${ }^{3}$ CK Shiu, FRCR, FHKAM (Radiology)

1 Department of Surgery, Union Hospital, Hong Kong

2 SH Ho Urology Centre, Department of Surgery, The Chinese University of Hong Kong, Hong Kong

congenital megacalycosis in paediatric patients are on the rise with the increasing use of imaging in antenatal screening. ${ }^{4}$ Although urinary stasis in the distended calyces may predispose to infection and stone formation, ${ }^{1,2}$ renal function remains normal and neither anatomic nor functional deterioration occur over time. ${ }^{5}$ The benign nature suggests that megacalycosis should be considered a condition rather than a disease, and the term "megacalycose" has been suggested as an alternative term by some authors. Treatment should target complications only. Identification of the condition is important to avoid unnecessary investigation and intervention in a normally functioning kidney despite the inherent anatomic defect. ${ }^{2}$
${ }^{3}$ Medical Imaging Department, Union Hospital, Hong Kong

* Corresponding author: chochaklam@yahoo.com.hk

\section{References}

1. Puigvert A. Le megacalice. J Urol Nephrol 1964;70:321-6.

2. Kimche D, Lask D. Megacalycosis. Urology 1982;19:47881.

3. Kalaitzis C, Patris E, Deligeorgiou E, et al. Radiological findings and the clinical importance of megacalycosis. Res Rep Urol 2015;7:153-5.

4. Kasap B, Kavukçu S, Soylu A, et al. Megacalycosis: report of two cases. Pediatr Nephrol 2005;20:828-30.

5. Redman JF, Neeb AD. Congenital megacalycosis: a forgotten diagnosis? Urology 2005;65:384-5. 